\title{
Numerical Investigation of Turbulent Flow over a Rotating Circular Cylinder under Marine Conditions
}

\author{
Khaled Alawadhi , Abdulwahab A. Alnaqi ${ }^{*}$ Jalal Alsarraf*, \\ Hasan A. Mulla ali ${ }^{*}$, Ahmad E. Murad* \\ *( Department of Automotive and Marine Engineering Technology, College of Technological Studies, The \\ Public Authority for Applied Education and Training, Kuwait Email: aa.alnaqi@paaet.edu.kw)
}

\begin{abstract}
Recent advancements in the field of computational fluid mechanics and the availability of high performance with regard to rotating software computing cylinders (RCs) have drawn attention to the field of flow accelerated corrosion. (FAC). Current studies aim to numerically predict turbulent flow characteristics around the rotating cylinder and the concomitant effects on the wall shear stresses and local mass fraction of inhibitors that are directly related to corrosion rate. This 3-D numerical investigation was carried out using the commercial CFX package from which the where SST turbulence model was selected to compute the unknown Reynolds stresses term in the incompressible and viscid form of the Navier-Stokes equation. The effect of three different cylinder rotation speeds and three brine temperatures on the wall shear stress and on brine mixing is reported. Results of the simulations revealed that both cylinder rotation speed and the temperature of the brine significantly affect wall shear stress and mixing of the inhibitor that in turn affects corrosion rate.
\end{abstract}

Keywords: Rotating cylinder, numerical model, corrosion, shear stress

\section{INTRODUCTION}

Corrosion processes can accelerate significantly under extreme environmental conditions such as high temperature, high pressure, and turbulent fluid flow. The turbulent flow at the RCE can bring material from the solution to the surface of the cylinder, and it can also carry material away from the surface. In the context of a corrosion study, the rate of mass transport to and from the metal surface is often the factor which governs the rate of corrosion [1].

RCEs are well suited to high mass transport studies in the turbulent flow regime [2]. A rotating cylinder causes turbulence due to entrainment of the fluid on the surface [3].

Few studies of turbulent flow physics around a rotating cylinder are available. Matthew and Klaus [4] compared turbulent flow characteristics in a straight pipe with corresponding behavior in a rotating cylinder. They made 3-D direct numerical simulations to understand the turbulent flow in these configurations and tried to formulate a procedure to transfer results from one configuration to another. S. Patankar [5] used Reynolds-averaged Navier-Stokes equations to formulate the turbulent flow caused by a rotating cylinder. They reported velocity field,volume fractions and distribution of inhibitor at various rotational speeds of the cylinder using 2-D numerical simulations.
In addition, the authors of [6] showed that in the fully turbulent layer, a logarithmic velocity profile exists, which is similar to that developed inside tubes and over flat plates. Another approximation employs the Reynolds Averaged Navier-Stokes (RANS) equations, which include turbulent viscosity by means of the $k-\varepsilon$ turbulent model $[7,8]$. In the current study, a turbulent flow prompted by a rotating cylinder is studied numerically using a 3-D computational domain identical to the setup used for the experimental study in [9]. A 3-D computational model is developed to understand the turbulent flow around the rotating cylinder. Commercial code ANSYS-CFX is used to solve the computational model. The novelty of the current numerical study is that the gas column above the water surface has been included in the computational domain to determine its effects on the turbulent flow characteristics. Thus, the current computational model can provide a better insight into the turbulent flow around a rotating cylinder.

\section{COMPUTATIONAL MODEL}

To analyze the fluid flow, continuity and Reynolds Averaged Navier-Stokes (RANS) equations were solved numerically using commercial code ANSYS-CFX. The appropriate set of equations with no source terms is given as follows [10], [11]:

$\frac{\partial}{\partial x_{i}}\left(u_{i}\right)=0$ 


$$
\begin{aligned}
& \rho \frac{\partial\left(u_{i}\right)}{\partial t}+\rho \frac{\partial\left(u_{i} u_{j}\right)}{\partial x_{j}}= \\
& \frac{\partial p}{\partial x_{i}}+\frac{\partial}{\partial x_{i}}\left(\mu\left(\frac{\partial u_{i}}{\partial x_{j}}+\frac{\partial u_{j}}{\partial x_{j}}-\frac{2}{3} \delta_{i j} \frac{\partial u_{j}}{\partial x_{i}}\right)\right) \\
& +\frac{\partial}{\partial x_{j}}\left(-\rho \overline{u_{i}^{\prime} u_{j}^{\prime}}\right)
\end{aligned}
$$

Where $p_{s} \rho_{v} f_{b}$ and $\mu$ are pressure, density, body force and dynamic viscosity respectively. $u_{j}$ and $u_{i}$ are velocity vectors for corresponding values of $i_{v} j=1,2,3$. The terms $\left(\frac{\partial}{\partial x_{j}}\left(-\rho \overline{u_{1} \dot{u}_{j}}\right)\right)$ that appeared in Eq. (2) are extra unknown terms and are referred to as Reynolds stresses [10]. Here $u i$ and $u^{\prime} i$ are fluctuating components of the velocity vectors $u i$ and $u j$ respectively. For the numerical closure of the RANS equations, it is necessary to solve unknown Reynolds stresses using an appropriate turbulence model. For the accurate prediction of shear stresses experienced by fluid in the vicinity of the rotating cylinder, accurate prediction of boundary layer, pressure losses, and flow separation within the boundary layer thickness is mandatory. Many researchers [12-14] have adopted the Shear Stress Transport (SST) turbulence model developed by Menter [15] and achieved accurate boundary layer prediction. Considering both computational economy and accuracy, the two equation $\mathrm{K}$ - omega SST turbulence model is consider to be one of the best turbulence models for rotating flow applications [16]. Based on the above discussion the SST turbulence model was adopted for the current numerical study. Details of the model can be found in [17].

\section{COMPUTATIONAL DOMAIN, MESH AND BOUNDARY CONDITIONS}

The computational domain for the current study consists of a cylindrical container filled with 0.8 litres of artificial sea water saturated with $\mathrm{CO}_{2}$ gas (brine) as shown in Fig. 1. All the bounded walls of the cylindrical container were modeled as no-slip adiabatic walls. The space between the upper wall brine was filled with air at a pressure of 1 bar and at $20^{\circ} \mathrm{C}$ for realistic flow predictions. A welded steel shaft was modeled as a rotating wall with no-slip conditions. Initially, with zero rotational speed of the steel shaft, a transient simulation for 20 minutes was set up in which an inhibitor was added to the upper surface of the sea water and was allowed to settle Simulations were then carried out for three rotational speeds of the steal shaft, i.e., 1000 RPM, 3000 RPM and 5000 RPM, in order to observe the flow characteristics and mixing of the inhibitor.

The computational domain was discretized with hexahedral volumes using ICEM-CFD. In order to fulfill the requirements of the SST turbulence model efficiently, Y-plus $\left(y^{+}\right)<1$ and the minimum number of nodes within the boundary layer should be 15 [11], topology of the mesh should consist of two O-grids as shown in Fig. 2. A mesh optimization study was carried out using four meshes, M1, M2, M3 and M4, varying the number of elements along $\mathrm{O}_{1}, \mathrm{O}_{2}$, height and circumference of the computational domain and the near wall node spacing. Details of all four meshes are listed in Table 1. All meshes were simulated using the same computational resources, i.e., 8 cores of a Core i7 $2.4 \mathrm{GHz}$ processor with $64 \mathrm{~GB}$ of available physical memory. Numerical results of average shear force on the shaft wall were compared for all numerical results for different meshes. It was found that the difference between the numerical results for average shear stresses for meshes M3 and M4 was less than $0.5 \%$ numerical results difference for average shear stress for mesh M3 and M4 falls within $0.5 \%$. Thus, based on its lower requirement for computational resources and computational time, mesh M3 was chosen for all numerical simulations.

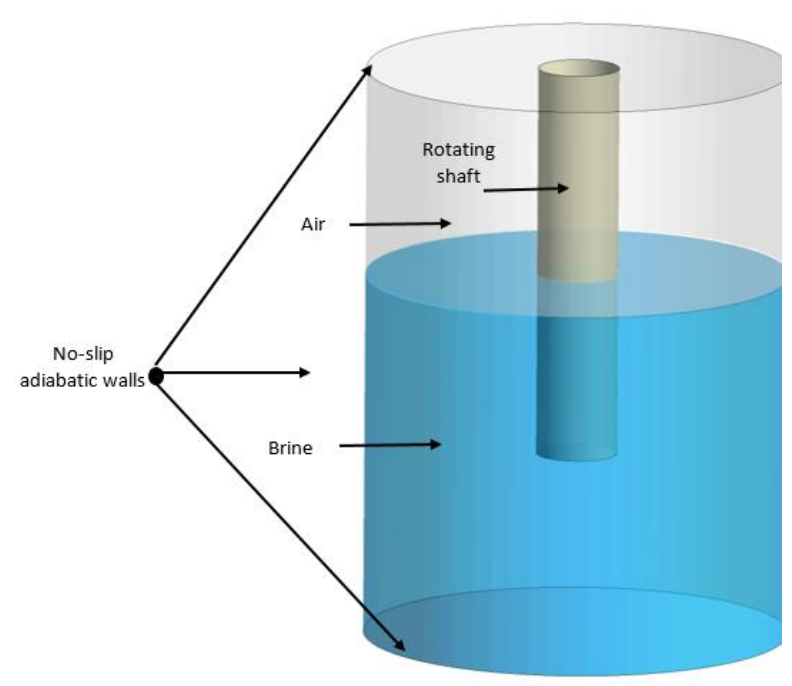

Figure 1. Computational domain for the numerical study 
Table 1: Details of all four meshes

\begin{tabular}{|c|c|c|c|c|c|c|c|c|c|}
\hline & $o_{1}$ & $\mathrm{O}_{2}$ & $4 \times N_{\text {ctrcum }}$ & $\mathrm{N}_{\mathrm{h}}$ & Near wall size & 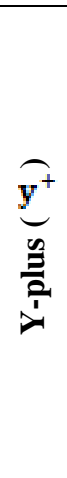 & 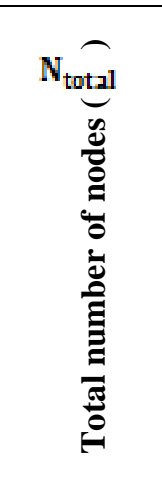 & 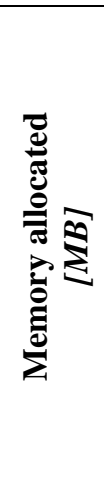 & 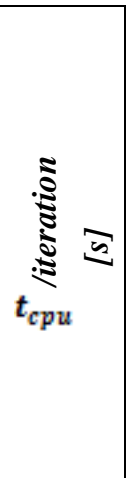 \\
\hline M1 & 20 & 30 & 80 & 100 & 0.2 & .98 & 400,000 & 2,735 & 22 \\
\hline M2 & 25 & 40 & 100 & 125 & 0.1 & 0.6 & 812,500 & 4,695 & 48 \\
\hline M3 & 30 & 50 & 120 & 150 & 0.05 & 0.4 & $1,440,000$ & 6,832 & 101 \\
\hline M4 & 50 & 65 & 150 & 175 & 0.02 & 0.3 & $2,327,500$ & 9,453 & 210 \\
\hline
\end{tabular}

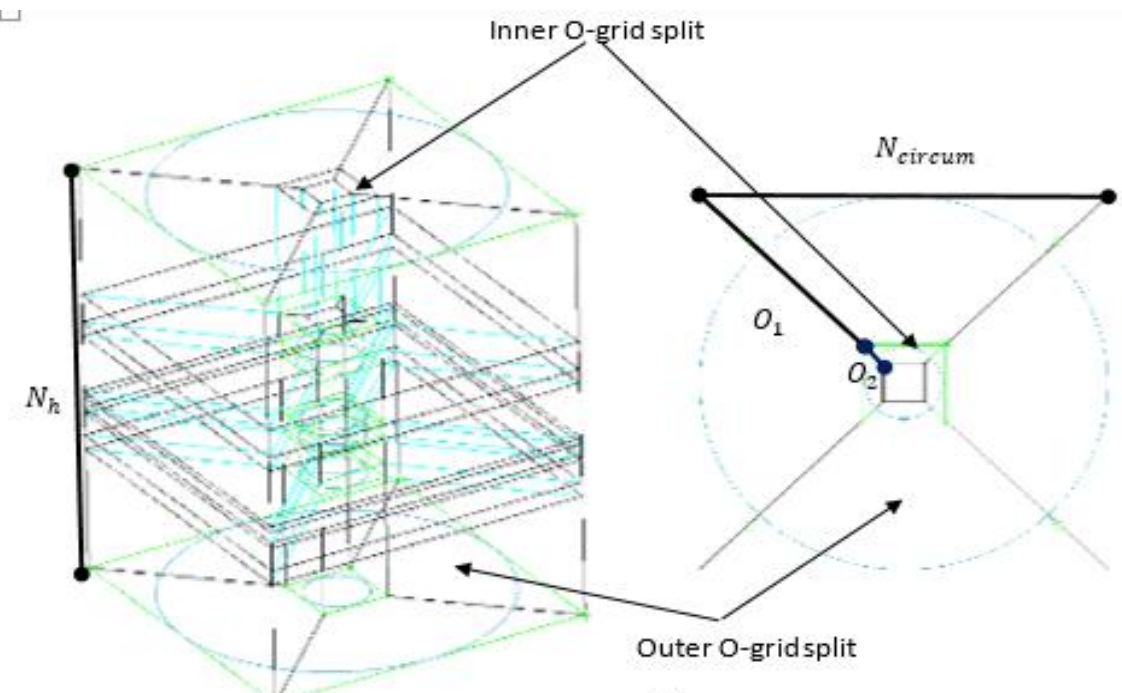

a)

b)

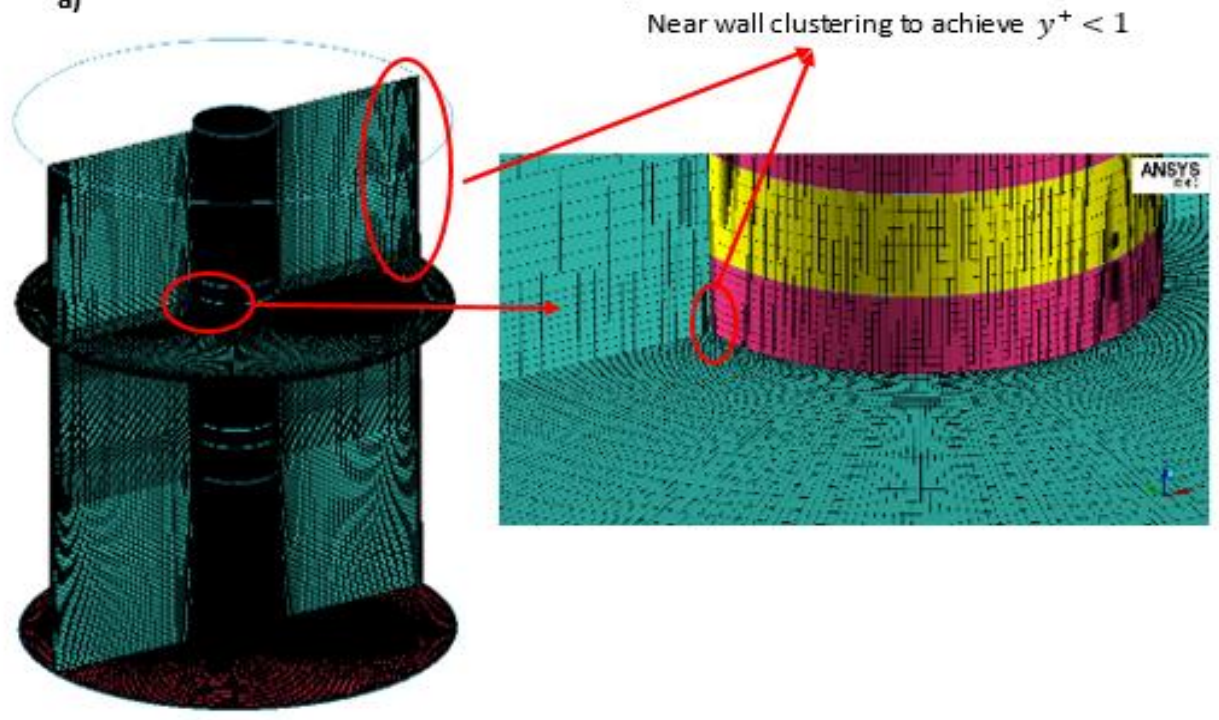

d)

c)

Figure 2. a) Mesh topology (isometric view), b) Mesh topology (top view), c) Mesh of the computational domain, d) Enlarged view of mesh near the rotating shaft 


\section{RESULTS AND DISCUSSION 4.1 Effect of rotational speed}

This study presents turbulent flow characteristics and patterns around a rotating shaft corresponding to different speeds of rotation. The simulation consists of two parts; 1) $30 \mathrm{ppm}$ of inhibitor was dropped onto the surface of the brine and allowed to settle in a simulation with total time of 20 minutes and time steps of 0.01 seconds while no angular speed was applied to the shaft; 2) results established in part 1 were used to initialize the simulations to observe flow characteristics at different shaft rotation speeds.

Fig. 3 shows the volume fractions of inhibitor and brine directly whereas the portion where volume fraction of brine and inhibitor is zero (blue portion) is contained by air. It is clear from Fig. 3 that, at the end of 20 minutes' solution time, the inhibitor settles in the form of a narrow strip with a maximum volume fraction value of 0.01 . It could be depicted in that strip portion volume fraction of brine is 0.99 and 1 all over below that narrow strip containing inhibitor.

Inhibitor Mass fraction
\begin{tabular}{|l}
$1.000 \mathrm{e}-002$ \\
$9.167 \mathrm{e}-003$ \\
$8.333 \mathrm{e}-003$ \\
$7.500 \mathrm{e}-003$ \\
$6.667 \mathrm{e}-003$ \\
$5.833 \mathrm{e}-003$ \\
$5.000 \mathrm{e}-003$ \\
$4.167 \mathrm{e}-003$ \\
$3.333 \mathrm{e}-003$ \\
$2.500 \mathrm{e}-003$ \\
$1.667 \mathrm{e}-003$ \\
$8.333 \mathrm{e}-004$ \\
$1.000 \mathrm{e}-015$
\end{tabular}

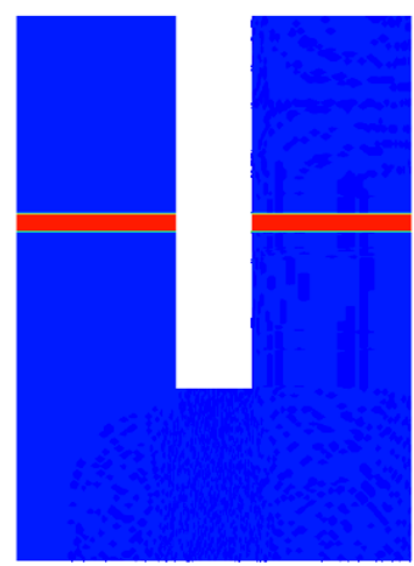

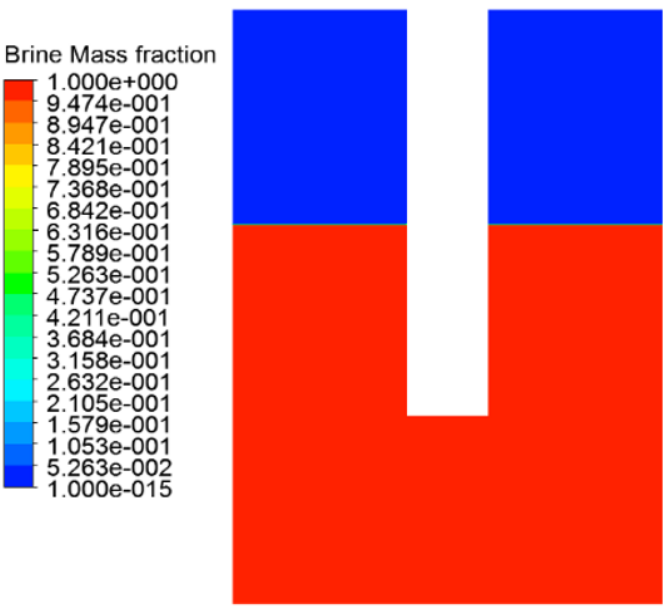

Figure 3. Inhibitor and brine mass fractions with no rotation speed of the cylindrical shaft

Figs. 4, 5 and 6 show volume fraction contours of the inhibitor, water and air mass fractions respectively at different RPM of the rotating cylinder. It can be seen from Fig.4 that mixing of the inhibitor increases with increasing rotational speed. Depicted from Fig. 4 that mixing of the inhibitor with rotational speed is increasing, while Figs. 5 and 6 suggest that with increasing rotational speed brine starts to rise up the side walls of the container due to centrifugal force. Meanwhile, air in the upper portion starts to penetrate downwards into the rotating cylinder. Penetration of the air increases with increasing rotational speed and is at a maximum for a rotational speed of 5000 RPM. This phenomenon can be seen clearly in the magnified views of Figs. 5 and 6.
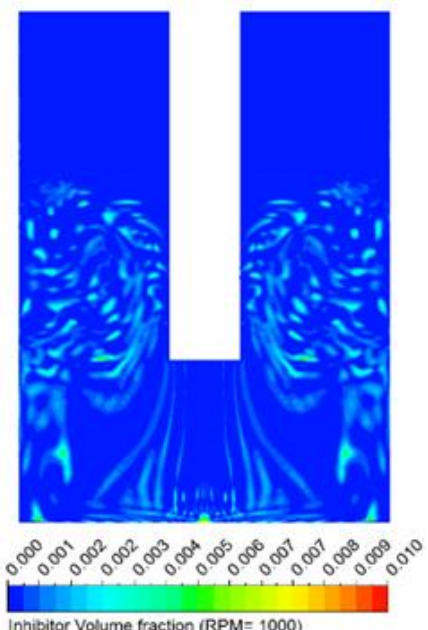
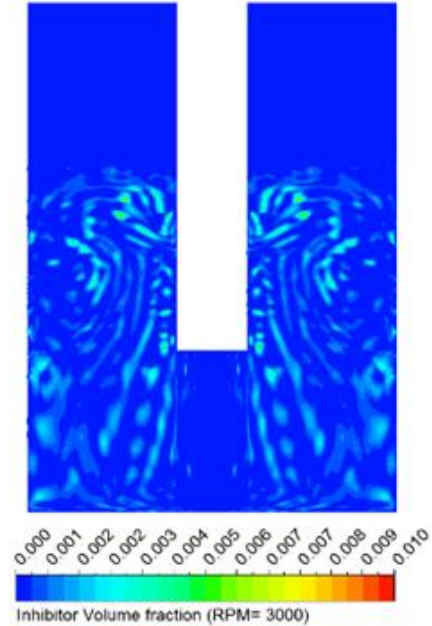
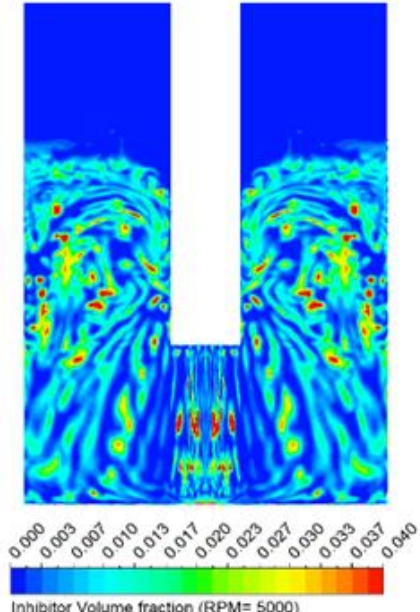

Figure 4. Volume fraction contours of inhibitor on the central plane corresponding to different rotational Speeds 

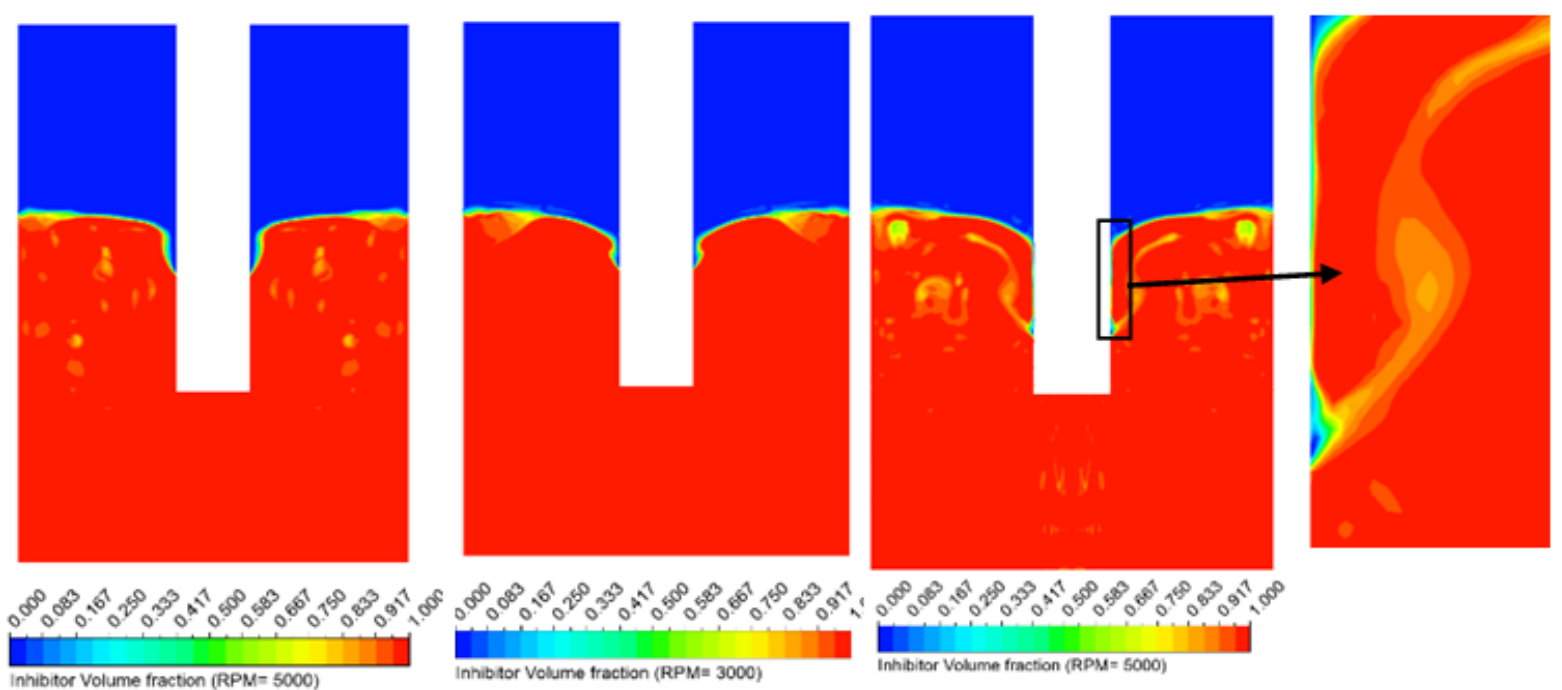

Figure 5. Volume fraction of brine on the central plane for different rotational speeds
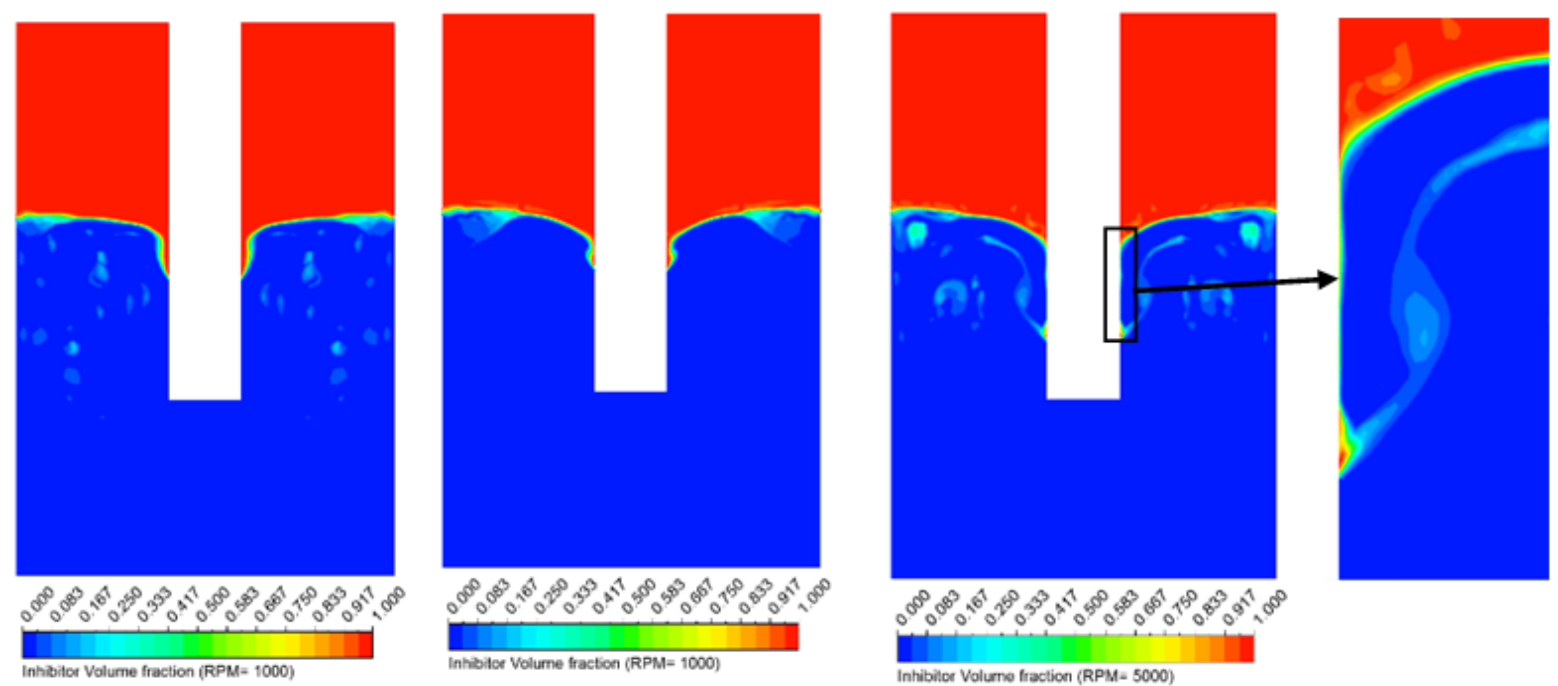

Figure 6. Volume fraction of air on the central plane for different rotational speeds

Fig. 7 shows the velocity vectors on the central plane. Four vortices can be seen in the vicinity of the rotating cylinder, caused by the motion of the cylinder. One vortex is located in the brine and the other three are in the air above the brine. Three vortices near the rotating cylinder are counter clockwise while the fourth one, away from the cylinder, in the upper corner, is clockwise and bigger in size.

Wall shear force has a significant role to play in flow accelerated corrosion. Fig. 9 shows the variation of wall shear stress along the rotating cylinder normalized height as shown in Fig. 8. Fig. 8 shows air volume fraction contours on the central plane and wall shear contours on the rotating cylinder wall. The magnitude of the wall shear stress increases with increasing speed of rotation (Fig. 9). In contrast, the total area of the cylinder on which the shear force of water is acting decreases with increasing speed of rotation due to penetration of the air along the rotating cylinder wall, as shown in Figs 5,6 and 8. The sudden decrease in the wall stresses corresponding to rotational speed 5000 RPM is again due to the change in the thickness of the air column around the rotating cylinder. It could be said, from the above discussion, that the value of the wall shear stress in the rotating cylinder is strongly dependent on the rotational speed and length of the immersed rotating cylinder in the brine solution. As can be seen from Fig. 3, whilst the cyclinder is stationary, almost $48 \%$ of its length is immersed in brine solution, but at rotational speeds of 1000, 2000 and 5000 RPM the immersed length of the rotating cylinder reduces to $47 \%, 33 \%$ and $14 \%$ respectively (Fig. 9). 

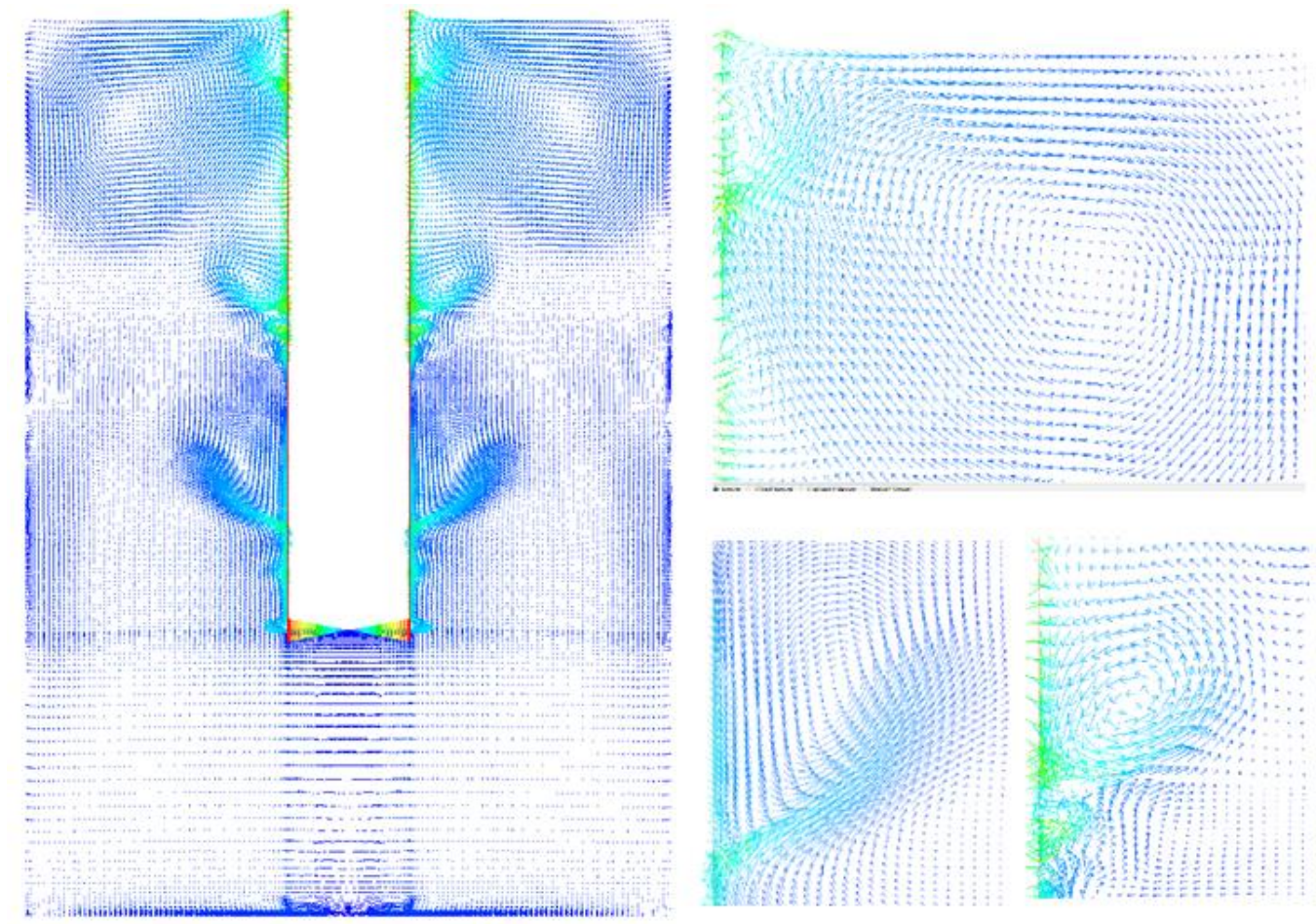

Figure 7. Velocity vectors at rotational speed of 5000 RPM
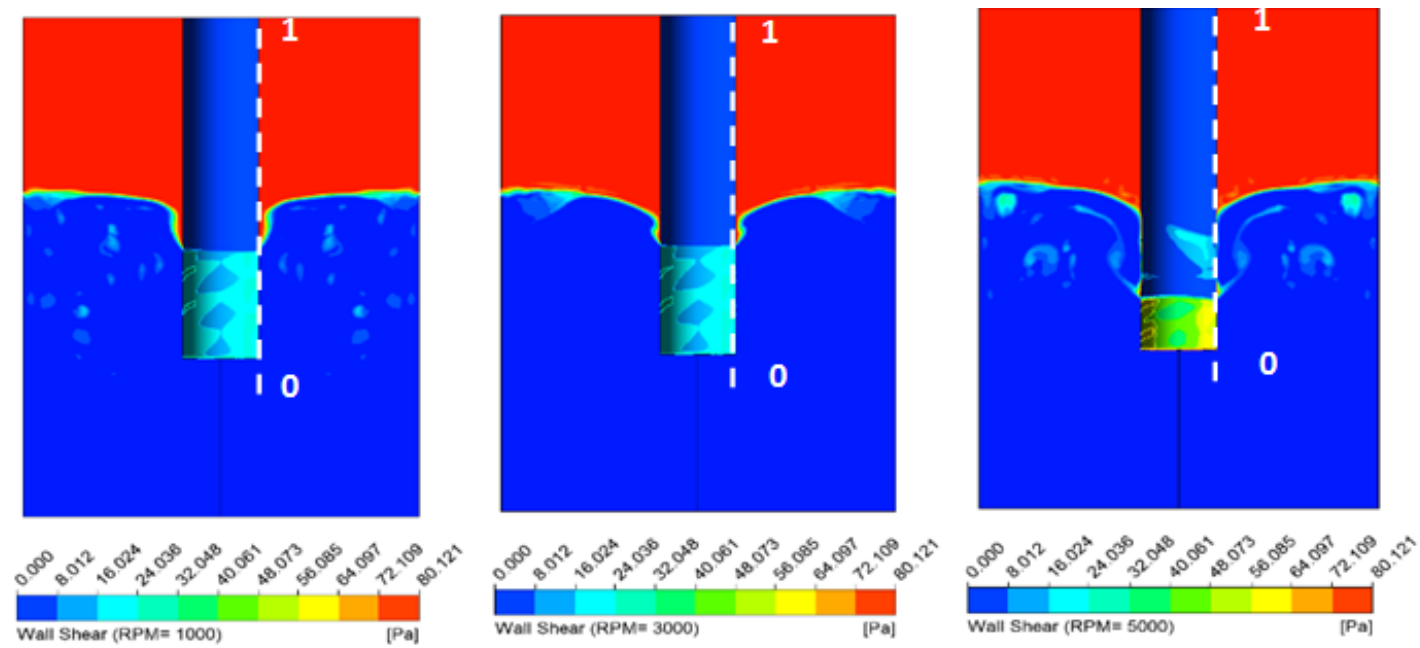

Figure 8. Wall shear stress contour in the cylinder and air volume fractions on the central plane

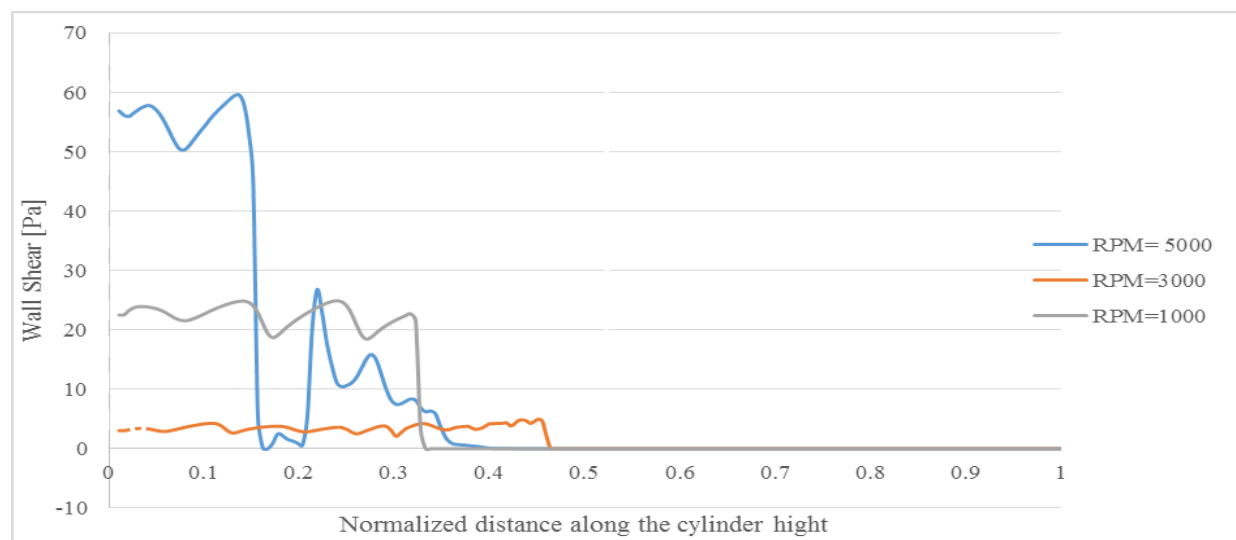

Figure 9. Wall shear stress along the normalized height of the cylinder for different rotational speeds 


\subsection{Effect of temperature}

In order to estimate the effect of temperature on the shear stress of brine solution and miscibility of inhibitor with the brine solution was predicted by modeling viscosity of the brine solution as a function of temperature. Each of three rotational shaft speeds, 1000 RPM, 2000 RPM and 5000 RPM, were simulated for three different initial temperatures of brine, $20^{\circ} \mathrm{C}, 50^{\circ} \mathrm{C}$ and $70^{\circ} \mathrm{C}$.

The variation in the viscosity of brine solution was modeled using an expression written in CEL (CFX Expression Language) as given by Eq. (3). The variation of viscosity with temperature is shown in Fig. 10. For all these simulations, atmospheric temperature was fixed at $25^{\circ} \mathrm{C}$ and heat transfer to the atmosphere was allowed through the top and side wall of the container.

$$
\mu=225.29 e^{-0.018 T}
$$

The variation in shear stresses on the rotating cylinder with temperature for different rotation speeds is shown in Figs. 11, 12 and 13. As discussed in the previous section, the magnitude of the shear stress increases with increasing speed of rotation but the effective area of application of force on the rotating cylinder reduces as penetration length of the cylinder decreases with increasing rotational speed. With this new set of boundary conditions, the same effect can be seen in Figs. 11, 12 and 13. It can also be observed in these figures that the temperature of a brine solution changes the immersed length of the rotating cylinder. A noticeable increase in the immersed length of the rotating cylinder can be observed in Figs. 11, 12 and 13 with increasing temperature at a fixed rotational speed. The effect of temperature is more prominent at higher rotational speeds. With an increase in the temperature of brine from $20^{\circ} \mathrm{C}$ to $50^{\circ} \mathrm{C}$ the immersed length increases from $10 \%$ to $17 \%$ of the total length of the rotating cylinder. With further increases in temperature to $70^{\circ} C_{x}$ the length of the immersed cylinder increases to $25 \%$. Thus, as the immersed length increases the total shear force acting on the rotating cylinder also increases and this leads to an increased corrosion rate. As discussed in the previous section, the length of the immersed section decreases with increased rotational speed as the brine solution tends to climb along the container walls due to viscous and centrifugal forces, and meanwhile, the air above the brine solution penetrates the brine along the walls of the rotating cylinder. This phenomenon decreases the immersed length of the rotating cylinder. With an increase in temperature, however, the viscosity of the brine decreases, so its tendency to rise along the outer walls of the container decreases. Consequently, penetration of air into the brine solution along the rotating cylinder wall decreases due to which the length of immersed rotating cylinder is greater at higher temperatures.

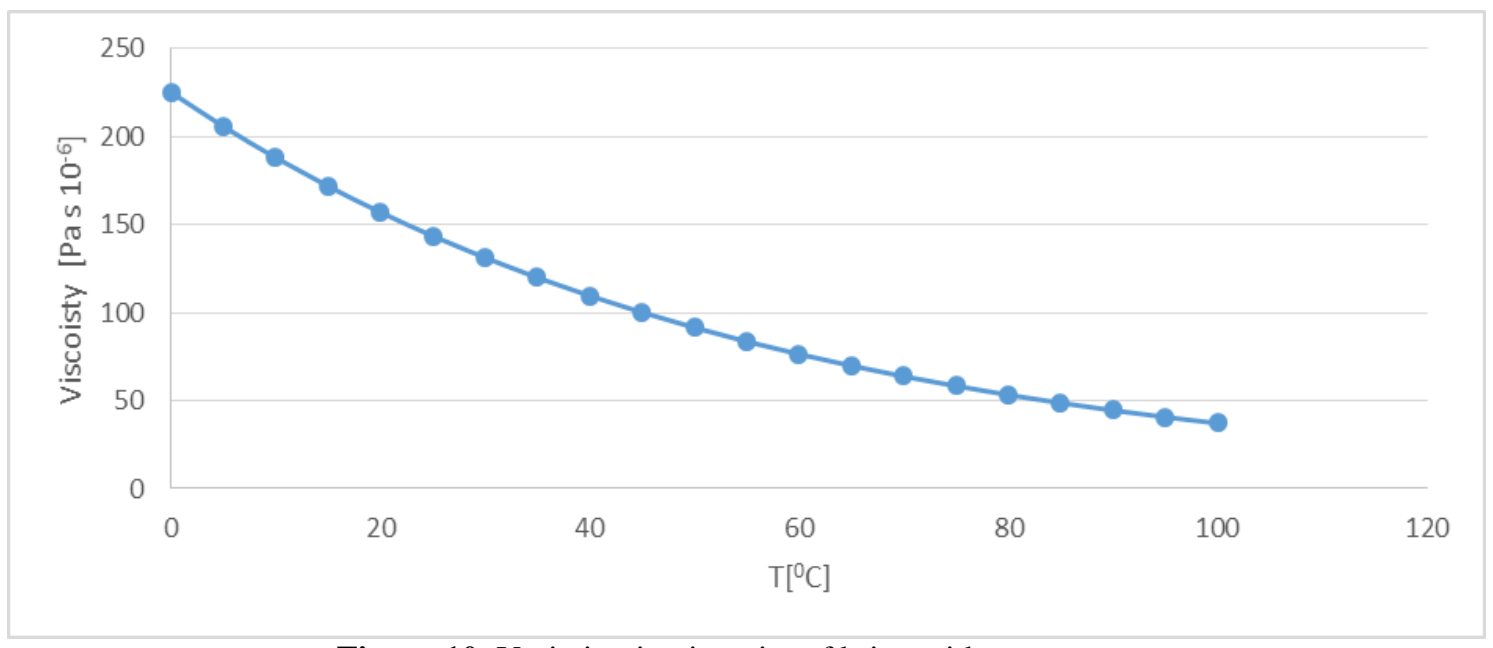

Figure 10. Variation in viscosity of brine with temperature 


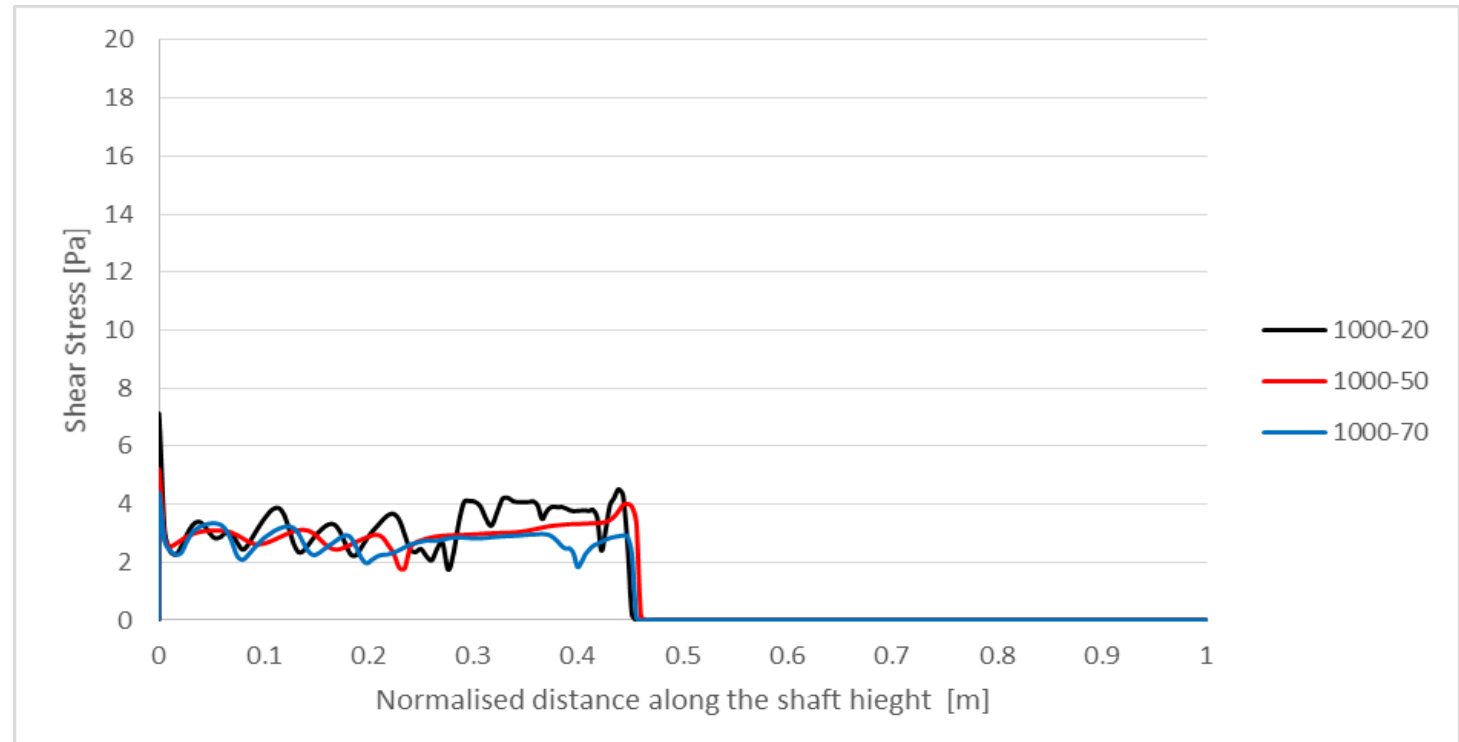

Figure 11. Variation in shear stress with temperature along the rotating cylinder wall at 1000 RPM

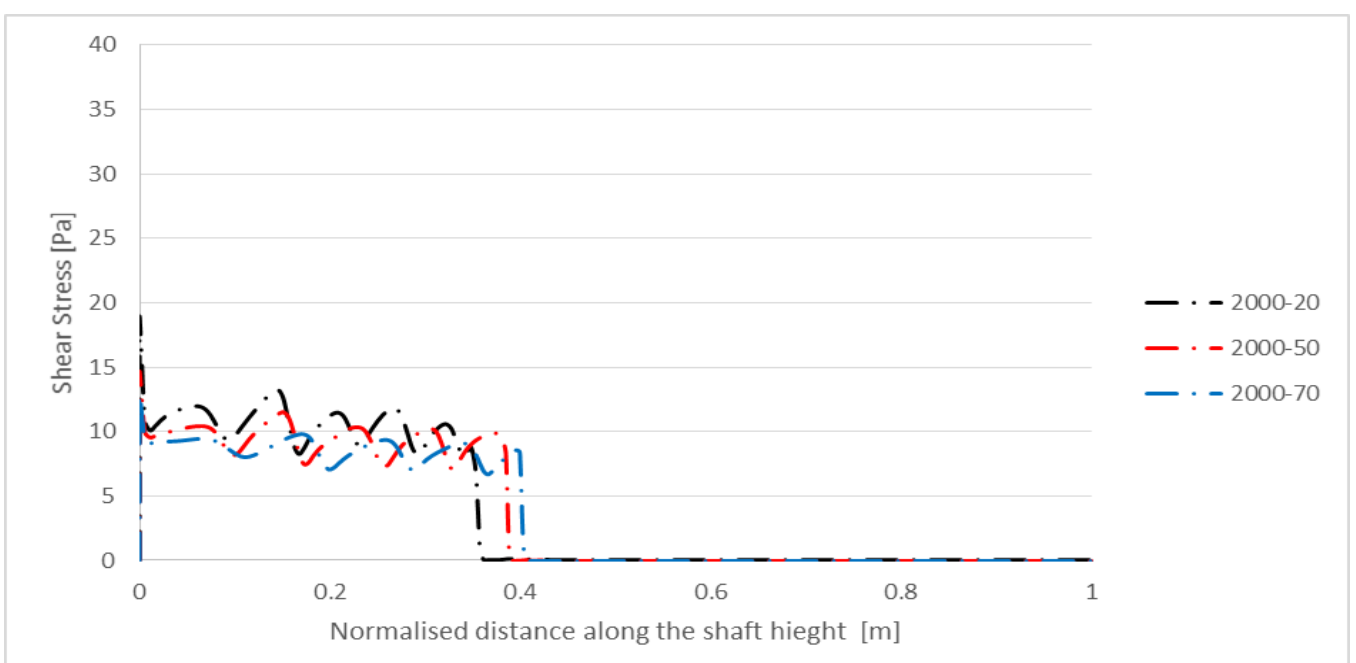

Figure 12. Variation in shear stresses with temperature along the rotating cylinder wall at 2000 RPM

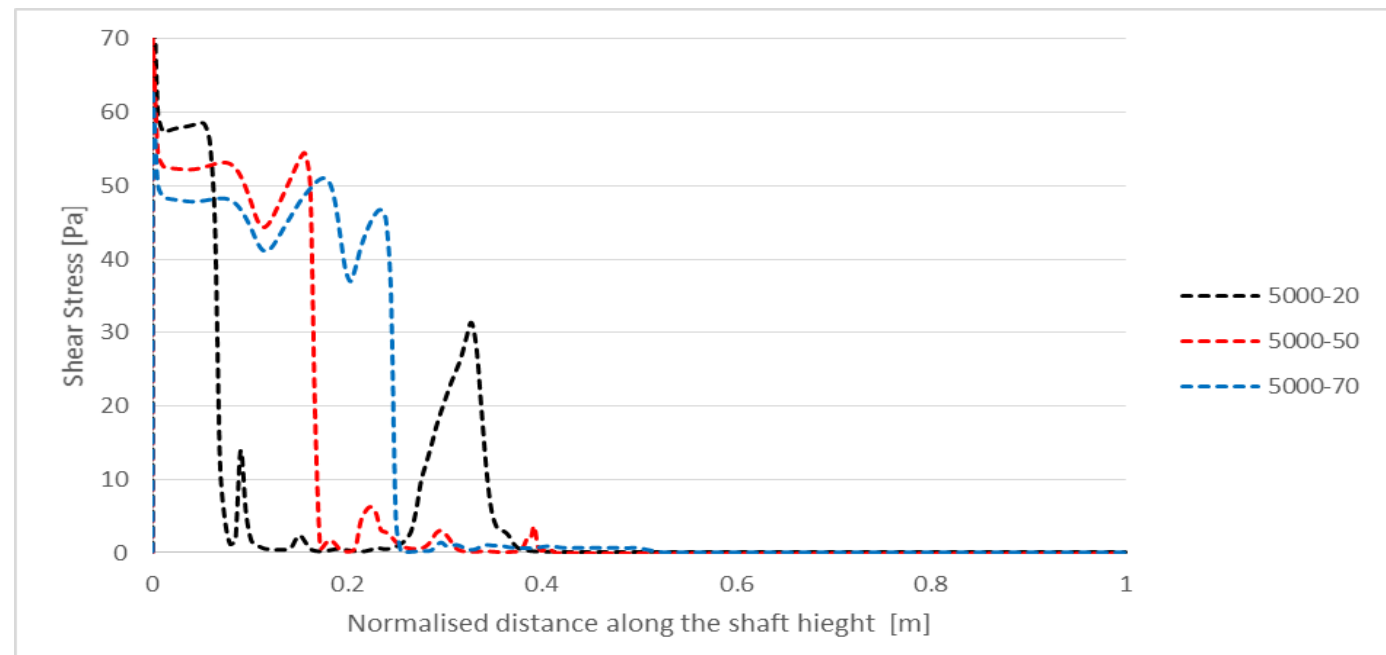

Figure 13. Variation in shear stresses with temperature along the rotating cylinder wall at 5000 RPM 


\section{CONCLUSION}

Shear stress on the rotating cylinder increases substantially with increasing rotational speed. Magnitude of maximum stress increases up to 8 times when rotational speed is increased from 1000 RPM to 2000 RPM and this increase becomes nearly $19 \%$ when rotational speed is increased to 8000 RPM.

With the increase in the rotational speed the area of contact between the brine solution and the rotating cylinder decreases with an increase in rotational speed due to penetration of air along the walls of the rotating cylinder. At a rotational speed of 5000 RPM, the area of contact reduces to $16 \%$ of the total area of the cylinder. An increase in the contact area leads to an increase in the total shear stress the rotating cylinder that may result in an increase in the corrosion rate as it would be very difficult for the inhibitor layer to remain intact on the rotating cylinder.

The contact area increases as the temperature of the brine solution increases. A $10 \%$ increase was computed when the temperature rose from $20^{\circ} C$ to $50^{\circ} C_{x}$ and an $18 \%$ increase when the temperature of the brine solution rose from $20^{\circ} \mathrm{C}$ to $70^{\circ} \mathrm{C}$.

\section{REFERENCES}

[1]. $\mathrm{CO}_{2}$ Corrosion in the Region Between the Static and Turbulent Flow Regimes

[2]. Lilian Raquel Moretto Ferreiraa*, Haroldo Araujo Ponteb, Luciana Schmidlin Sanchesb, Ana Carolina Tedeschi Gomes Abrantesb aCentro Universitário Tupy - UNISOCIESC, Joinville, SC, Brazil bLaboratório de Eletroquímica de Superfícies e Corrosão, Universidade Federal do Paraná - UFPR, Curitiba, PR, Brazil Received: March 9, 2014; Revised: March 4, 2015.

[3]. J. M. Grau and J. M. Bisang, J. Appl. Electrochem., 36 (2006) 759.

[4]. 4. M. A. Carlos, M. F. Leonardo, R. C. N. Shirley, P. Y. Dario, V. Q. Custodio, Scietia Et Technica (2007) 151.

[5]. H. K. Versteeg and W. Malalasekera, "An Introduction to Computational Fluid Dynamics - The Finite Volume Method," Fluid flow handbook. McGraw-Hill. p. 267, 1995.

[6]. S. Patankar, "Numerical heat transfer and fluid flow," Series in computational methods in mechanics and thermal sciences. pp. 1197, 1980.

[7]. 17. J. Y. Hwang, K. S. Yang, D. H. Yoon and K. Bremhorst, Int. J. Heat Fluid Fl., 29 (2008) 1268.

[8]. E. P. Rivero, P. Granados, F. F. Rivera, M. Cruz and I. González, Chem. Eng. Sci., 65 (2010) 3042.

[9]. J. A. Delgadillo, R. Enciso, C. Ojeda, I. Rodríguez, Int. J. Electrochem. Sci., 7 (2012) 2065.

[10]. K. Alawadhi and M. J. Robinson, "Preferential Weld Corrosion of X65 Pipeline Steel in Flowing Brines Containing Carbon Dioxide," Corros. Eng. Sci. Technol., vol. 46, no. 4, pp. 318-329, 2011.

[11]. H. K. Versteeg and W. Malalasekera, "An Introduction to Computational Fluid Dynamics - The Finite Volume Method," Fluid flow handbook. McGraw-Hill .... p. 267, 1995.

[12]. S. Patankar, "Numerical heat transfer and fluid flow," Series in computational methods in mechanics and thermal sciences. pp. 1197, 1980.

[13]. X. Cai, R. Gu, P. Pan, and J. Zhu, "Unsteady aerodynamics simulation of a full-scale horizontal axis wind turbine using CFD methodology," Energy Convers. Manag., vol. 112, pp. 146-156, 2016.

[14]. M. A. Sayed, H. A. Kandil, and A. Shaltot, "Aerodynamic analysis of different windturbine blade profiles using finite-volume method," Energy Convers. Manag., vol. 64, pp. 541-550, 2012.

[15]. N. N. Sörensen, J. a. Michelsen, and S. Schreck, "Navier-Stokes predictions of the NREL phase VI rotor in the NASA Ames 80 $\mathrm{ft} \times 120 \mathrm{ft}$ wind tunnel," Wind Energy, vol. 5, no. 2-3, pp. 151-169, 2002.

[16]. F. R. Menter, “AIAA 93 - 2906 Zonal Two Equation k · co Turbulence Models for Aerodynamic Flows. Mailing Address: 24th Fluid Dynamics Conference FOR AERODYNAMIC FLOWS," 1993.

[17]. T. Wang, "A brief review on wind turbine aerodynamics," Theor. Appl. Mech. Lett., vol. 2, no.6, p. Article 062001, 2012.

[18]. "ANSYS 16, ANSYS-CFX user manual". 